\title{
Calculation of propeller's load noise using LES and BEM numerical acoustics coupling methods
}

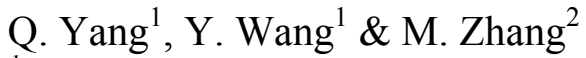 \\ ${ }^{1}$ College of Naval Architecture and Marine Power, \\ Naval University of Engineering, Wuhan, China \\ ${ }^{2}$ College of Electronic Engineering, Naval University of Engineering, \\ Wuhan, China
}

\begin{abstract}
Coupling large eddy simulation (LES) and boundary element numerical acoustics methods in the frequency domain to predict the underwater noncavitation far-field load noise in the ship wake flow, which presents a new method to measure the propeller noise level in the ship engineering. The Dynamic Smagorinsky-Lilly model (DSM) is applied to subgrid-scale (SGS) stress tensor in the process of unsteady field LES simulation. Then the sound pressure field is determined solely by the pressure distribution and normal velocity distribution on the closed surface including blades and hub surface after node numbers conservative transfer of the acoustic nodal sources to the acoustic grid. The predicted results show that the steady thrust and torque coefficients of the propeller differ by less than $2 \%$ on design point to the experiment data, and the pressure coefficients fit very well. The unsteady calculation can present precisely the blade passing frequency (BPF), its harmonics and wake axis passing frequency (APF) information. Under low frequency, the concentrated area contributing mainly to the noise lies in the blade tip and trailing edge near the root section, whereas for high frequency the source intensity transfers to the blade surface with a centralized pressure load. The spherical surface sound pressure distribution and wake point frequency spectra line of the propeller just get quantitative agreement with the reference calculated results because of the slight difference of ship wake distribution, and the calculated total sound pressure level below $200 \mathrm{~Hz}$ is $126.4 \mathrm{~dB}$.

Keywords: propeller, load noise, boundary element method, integral method, frequency domain.
\end{abstract}




\section{Introduction}

The principle mechanisms of underwater radiated noise of the ship propeller can be divided into four parts, thickness noise, which dues to the displacement of the water by the blade profile, steady load noise, which points to the pressure difference between the suction and pressure sides of the blade when they are rotating, unsteady load noise, which caused by the periodic fluctuation and the random vibration of the blades induced by spatially non-uniform wake flow behind the vessel, and the broadband spectrum, which associates with the interaction noise between the turbulent inflow and the leading edge of the blades, the trailing edge vortex shedding noise and cavitation radiated noise caused by the bubbles collapse process [1-3]. The radiated noise of non-cavitating propeller in a uniform flow condition mainly consists of monopole thickness noise and dipole Gutin sound [3], and as for the ship propeller, the Gutin sound often contributes little to the overall noise in the far field and can be negligible [1]. Whereas in a ship wake condition, the dominating source comes from unsteady blade loading acting as a dipole source, or named as load noise directly $[4,5]$.

In the phases of the propeller design process nowadays, it general uses the empirical wake fraction to get the optimum circulation distribution, then aims for the highest possible level hydrodynamic performances, efficiency, for instance. However, the effects of the real wake flow and its effects on the radiated noise haven't been involved into the design loop $[6,7]$. So the investigation into the acoustic performances for a propeller working in a spatially non-uniform wake flow will be productive for the hull-behind low noise propeller design focusing on its acoustics target directly.

For flows with a relatively low Mach number, propeller operation, for instance, feedback effects of the sound to the source fluctuated flow is negligible in general, and the turbulent quadrupole noise source can be neglected too comparing with the rotating load noise [8]. It is thus possible to predict the farfield radiated noise of the propeller by the weak coupling method, or named as hybrid CFD/CA method, of using computational fluid dynamics (CFD) to predict the source flow field and computational acoustics (CA) to analyze the resulting acoustical field. As a simplification, the CFD calculation to obtain the timedomain fluctuating pressure on the blades can be included in the hypothesis of incompressibility due to the high Reynolds number and low Mach number for the flow, while the retarded time effects due to the finite sound velocity must be accounted for in the propagation $[9,10]$.

To get an accurate prediction of the local unsteady noise sources, available CFD techniques includes the unsteady Reynolds-Averaged Navier-Stokes (URANS), Scale-Adaptive Simulation (SAS), Detached Eddy Simulation (DES), and Large Eddy Simulation (LES) for the engineering in general, and their ability seems to promise gradually [11]. The most practical applications for the radiated noise prediction in the marine engineering are surface integrals method in the time domain based on Ffowcs Williams-Hawkings equation (FWH), including both of the porous FWH equation and Kirchhoff FWH equation [2, 4, $5,9,10]$. The time domain method must work by time series, and at least 
including the points shifting in a whole circle. A frequency domain method that aims to predict the harmonic acoustic intensity directly replaces the rotating source distribution on a propeller blade with a stationary distribution over the propeller disc, which has the advantage of eliminating the rotational component of the source motion from the calculations. So the moving source distribution as a function of time can be replaced by its Fourier series as a function of azimuth and the acoustic modes is introduced into solving that is easy to be analyzed [10]. As for the unbounded exterior acoustic calculation, the direct boundary element method (DBEM) in frequency domain can be a good selection for the applications [12, 13].

Seol et al. $[4,5]$ used the separate computations of the flow field being analyzed with non-viscous potential-based panel method and the noise propagation being predicted using time-domain FWH equation (Farassat formulation 1A) to investigate the no-skewed DTRC4119 propeller's noncavitation and DTMB4381 propeller's blade sheet cavitation overall noise and the thickness and load noise components contribution and their noise directivity characteristics. The results showed that thickness noise was much smaller than the load noise component under the non-cavitation condition. Considering the determination of the source flow field for noise prediction and the limit of panel method, the attention in this paper is to enhance the precision of the viscous flow field prediction as high as possible, so the most promising LES simulation is adopted to predict the same 4119 propeller flow in a wake flow, and then the source fluctuations are fed to the BEM numerical acoustical computation as input data, which aims to demonstrate the capability of the new approach for propeller noise judging.

The LES and BEM numerical acoustics theory will be presented in section 2 . The unsteady flow field calculation and the acoustic prediction will be completed in section 3. Section 4 will summarize the results that have been obtained in this study.

\section{LES and BEM acoustics methodology}

Fig. 1 shows the general solving steps and typical available hybrid methods for the flow noise and propeller noise prediction. Herewith, $\Omega_{F}$ denotes the flow area calculational domain and confined by $\Gamma_{F}$ where the acoustic sources are interpolated from the fluid simulation to the acoustic computation. Subscripts $\mathrm{S}$, F, and A denote the source, flow and acoustics respectively. Fig. 2 presents the common schematic for the flow-induced noise coupled computation in time and frequency domain, and the sequentially coupled approach in frequency domain is adopted in this paper.

LES is currently widely used for solving the turbulent fluids required for acoustic analogy computations. In the LES method the large scales are directly resolved and the effect of the small scales on the large scales are modeled by the sub-grid scale (SGS) model, and the turbulent models used in the RANS simulation are not needed again. At the same time, it requires a relatively high computational cost, and the mesh space and time scale's requirement is also the 
88 Boundary Elements and Other Mesh Reduction Methods XXXIII

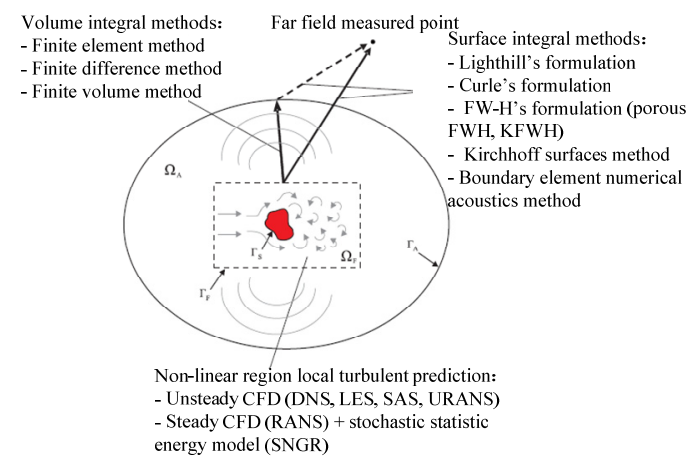

Figure 1: The general solving steps and typical available hybrid methods for the flow noise prediction.

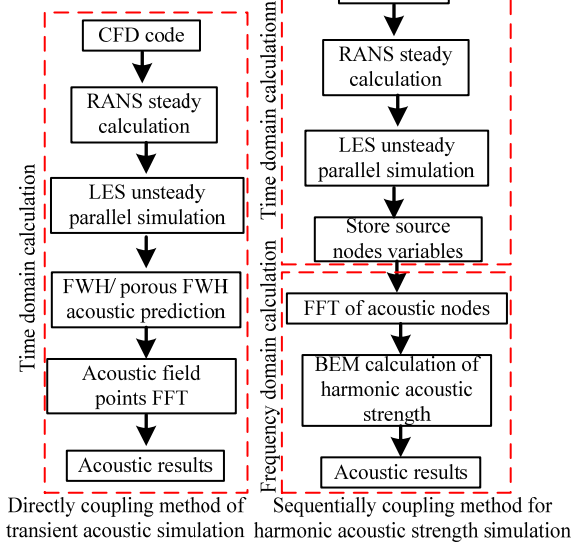

Figure 2: $\quad$ The solving loop for flow-acoustics hybrid prediction.

highest. It is obvious that the spatial filtering function of the incompressible flow equations to define the large eddy and the subgrid modelling problem mainly affect the predict errors $[14,15]$. Reference to the LES simulation construction for propeller crashback in Vysohlid Ph.D dissertation (2007), the dynamic Smagorinsky model with modification by Lilly (1992) (DSM-Lilly) is used as the SGS in this paper, and it reads

$$
\tau_{i j}=\frac{1}{3} \delta_{i j} \tau_{k k}-2 \mu_{t} \bar{S}_{i j}
$$

where $\delta_{i j}$ is the Kronecker symbol, $\tau_{k k}$ is isotropic SGS stress, $\mu_{t}$ is the SGS turbulent viscosity and $\bar{S}_{i j}$ the large-scale strain rate tensor.

$$
\mu_{t}=(C \Delta)^{2}|\bar{S}|, \quad \bar{S}_{i j}=\frac{1}{2}\left(\frac{\partial \bar{u}_{i}}{\partial x_{j}}+\frac{\partial \bar{u}_{j}}{\partial x_{i}}\right), \quad|\bar{S}|=\sqrt{2 \bar{S}_{i j} \bar{S}_{i j}}
$$


where $C$ is the Smagorinsky coefficient, which adjusts automatically to the flow type corresponding to the size of the test-filtered width. The local grid size $\Delta$ is one third of the element volume.

After the fluctuated pressure and normal velocity distribution on the blades in the time series has been obtained by the CFD calculation, the weighted interpolation transfer of the CFD nodes to the acoustic nodes should be done to map the input data of the BEM calculation. The variables transformation will get a little effect on the source strength. So the mapping is commonly completed between the same grids if the computer allowed, called one to one transformation. If the BEM grids are too much, counter-refinement of the acoustic nodes to some extent is also ok, named many to one, and the weighted interpolation is needed for this case of course [12,13]. For the 4119 propeller is no-skewed and is relatively easy to make the mesh topology to control the mesh density, so the one to one model is chosen in this paper.

After accounting for the effects of blades rotating, the wave equation changes to

$$
\frac{\partial^{2} \rho^{\prime}}{\partial t^{2}}-c_{0}^{2} \frac{\partial^{2} \rho^{\prime}}{\partial x_{i}^{2}}=\frac{\partial^{2} T_{i j}}{\partial x_{i} \partial x_{j}}+\frac{\partial f_{s}}{\partial x_{i}}+\frac{\partial m_{s}}{\partial t}
$$

where $\rho$ relates to the acoustic component of the density, $c_{0}$ is the sound speed, and $T_{i j}$ is the Lighthill stress tensor that reads

$$
T_{i j}=\rho v_{i} v_{j}+\left(p^{\prime}-c_{0}^{2} \rho^{\prime}\right) \delta_{i j}-\sigma_{i j}
$$

where $\rho v_{i} v_{j}$ is the instantaneous Reynolds stress, $\sigma_{i j}$ is the viscous stress term that is generally negligible because of its extremely inefficient octupole nature as a noise source. The second term is also often thought to be small in absence of strong temperature inhomogeneities, the propeller flow for example. So, as for the low Mach number flow, the equation (1) can be simplified into

$$
T_{i j} \approx \rho v_{i} v_{j}
$$

In the CFD solving process, the variable $T_{i j}$ and pressure and three velocity components are all used as residuals monitor, so that it can be sure that the convergence is completed. But the $T_{i j}$ term refers to the quadrupoles is neglected in the acoustic calculation $[4,5,9,10]$. And so does the third $\frac{\partial m_{s}}{\partial t}$ term too that is equivalent to monopole thickness noise. The remained $\frac{\partial f_{s}}{\partial x_{i}}$ term in equation (2) is just the main non-cavitation load noise and will be analyzed in this paper.

When using the BEM numerical acoustic method in the frequency domain for the propeller, the variable $f_{\mathrm{s}}$ points to the fluctuated pressure on the blades and hub surface. Specifically, the exterior propeller radiated noise problem (see Fig. 3) can be solved by the DBEM equation [13],

$$
C(\vec{r}) \cdot p(\vec{r})=\int_{\Omega_{a}}\left(p\left(\vec{r}_{a}\right) \cdot \frac{\partial G\left(\vec{r}, \vec{r}_{a}\right)}{\partial v}+j \rho \omega G\left(\vec{r}, \vec{r}_{a}\right) \cdot v_{v}\left(\vec{r}_{a}\right)\right) d \Omega\left(\vec{r}_{a}\right)
$$


where $C(\mathbf{r})=\left\{\begin{array}{l}1, \mathbf{r} \in \mathbf{V}, \\ 1 / 2, \mathbf{r} \in \Omega_{a} \text { is the sound pressure coefficient, } V \text { is the volume of the } \\ 0, \vec{r} \notin \mathbf{V} .\end{array}\right.$ closed domain for surface integral, $p(\vec{r})$ is the sound pressure at position $\vec{r}, p\left(\vec{r}_{a}\right)$ is the pressure distribution on the closed surface, $G\left(\vec{r}, \vec{r}_{a}\right)=\frac{e^{-j k\left|\vec{r}-\vec{r}_{a}\right|}}{4 \pi\left|\vec{r}-\vec{r}_{a}\right|}$ is the Green's kernel function, $v$ denotes for the normal direction of the closed surface $\Omega_{a}$ with a positive orientation into the unbounded domain $(v=-\vec{n})$, and $v_{v}\left(\vec{r}_{a}\right)$ is the normal velocity component on position $\vec{r}_{a}$, see Fig. 4 . Hence, the sound pressure $p(\vec{r})$ for any position $\vec{r}$ outside the closed surface $\Omega_{a}$ in the unbounded domain can by solely determined by the $p\left(\vec{r}_{a}\right)$ and $v_{n}\left(\vec{r}_{a}\right)$ on the $\Omega_{a}$ surface. The $\Omega_{a}$ surface for the propeller is shown in Fig. 3.

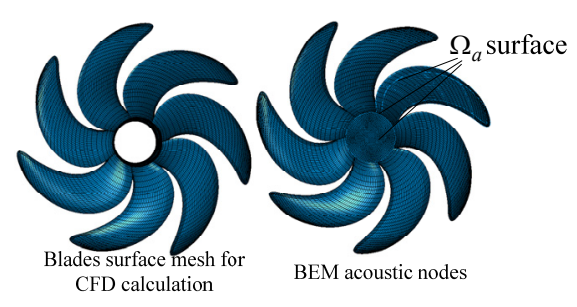

Figure 3: The CFD mesh and acoustics nodes.

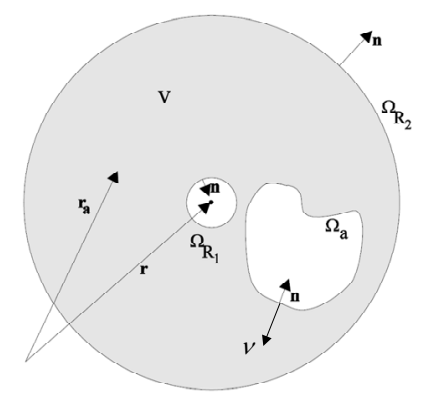

Figure 4: DBEM integral formulation variables definition for unbounded domain [13].

\section{Propeller noise numerical simulation}

This section will present the non-cavitation load noise of the DTRC4119 using the coupling method interpreted above, and do the qualitative and quantitative compare with the references [4] and [5], so as to validate indirectly the ability of the coupling method using in propeller non-cavitation noise prediction. 


\subsection{LES simulation of the unsteady flow field}

To satisfy the requirement of the smallest mesh size for LES simulation, and quicken the solution, special care should be paid to the mesh creation for the propeller to get the mesh quality as high as possible, high determinant, for instance, especially for the blade leading and trailing edge and their vicinity and the tip area geometry. Fig. 5 shows the propeller CAD geometry and hex mesh topology structure adopted for LES simulation at last and the local surface mesh refinement details, and the blade leading edge, trailing edge, and tip area are associated and refined by independent blocks. The O-grid topology around the blade surface wall is used to capture the boundary layer flow structure through to the blades. The mesh determinant, which can be a comprehensive mesh quality index, also is shown in Fig. 5. A determinant value of 1 will indicate a perfectly regular hex/tet mesh element, 0 will indicate an element degenerate in one or more edges, and negative values will indicate inverted elements, which are not allowed in the commonly used commercial CFD solvers. To the best of author's experience [18-20], when the smallest mesh determinant value is bigger than 0.2 , the solving convergence will be good and fast. If the reasonable mesh topology, good turbulence model and effective wall function (the first node adjacent to the wall should be fit to the limit of $y+$ value) are added in addition, it will get a promising result for the RANS simulation.

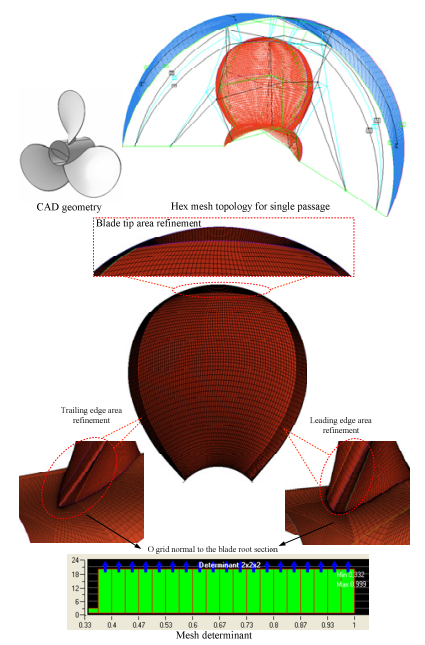

Figure 5: $\quad$ DTRC4119 propeller CAD geometry and hex mesh topology.

Mesh sensitive analysis is an essential step in the CFD calculation process. Fig. 6 shows different mesh topologies used in validation of the hydrodynamics of DTRC4119 propeller. The calculated open water thrust and torque coefficients of the mesh series are shown in Table 1, and the pressure coefficient distribution at $0.7 R$ section is shown in Fig. 7 for the G3 and G4 mesh. According to Table 1 and Fig. 7, it is obvious that the G4 mesh topology and grid density is optimum and being the prototype for the LES simulation shown in Fig. 5. 


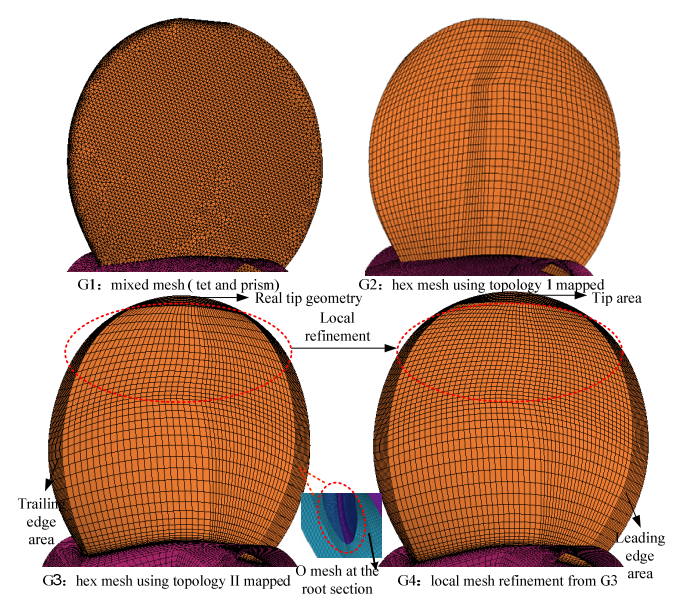

Figure 6: Different mesh topologies construction for propeller 4119.

Table 1: Mesh sensitive analysis for RANS simulation of DTMB4119 propeller.

\begin{tabular}{|c|c|c|c|c|c|c|c|}
\hline$J$ & Mesh & $K_{\mathrm{t} \_\mathrm{Cal}}$ & $K_{\mathrm{t} \_}$Exp & Error(\%) & $10 K_{\mathrm{q} \_} \mathrm{Cal}$ & $10 K_{\mathrm{q} \_} \operatorname{Exp}$ & Error $(\%)$ \\
\hline \multirow{3}{*}{0.5} & G1 & 0.2775 & 0.285 & -2.617 & 0.4583 & 0.477 & -3.923 \\
\hline & G2 & 0.2858 & 0.285 & 0.289 & 0.4615 & 0.477 & -3.245 \\
\hline & G4 & 0.2858 & 0.285 & 0.281 & 0.4615 & 0.477 & -3.249 \\
\hline \multirow{3}{*}{0.7} & G1 & 0.1998 & 0.2 & -0.119 & 0.3524 & 0.36 & -2.105 \\
\hline & $\mathrm{G} 2$ & 0.2035 & 0.2 & 1.755 & 0.3522 & 0.36 & -2.170 \\
\hline & G4 & 0.2035 & 0.2 & 1.750 & 0.3521 & 0.36 & -2.194 \\
\hline \multirow{3}{*}{0.833} & G1 & 0.1484 & 0.146 & 1.637 & 0.2827 & 0.28 & 0.964 \\
\hline & $\mathrm{G} 2$ & 0.1510 & 0.146 & 3.415 & 0.2797 & 0.28 & -0.106 \\
\hline & G4 & 0.1479 & 0.146 & 1.301 & 0.2824 & 0.28 & 0.857 \\
\hline \multirow{3}{*}{0.9} & G1 & 0.1213 & 0.12 & 1.077 & 0.2456 & 0.239 & 2.746 \\
\hline & G2 & 0.1246 & 0.12 & 3.857 & 0.2429 & 0.239 & 1.615 \\
\hline & G4 & 0.1184 & 0.12 & -1.333 & 0.2401 & 0.239 & 0.460 \\
\hline \multirow{3}{*}{1.1} & G1 & 0.0329 & 0.034 & -3.191 & 0.1114 & 0.106 & 5.121 \\
\hline & $\mathrm{G} 2$ & 0.0420 & 0.037 & 13.581 & 0.118 & 0.106 & 11.420 \\
\hline & G4 & 0.0318 & 0.033 & -3.636 & 0.108 & 0.106 & 1.859 \\
\hline
\end{tabular}



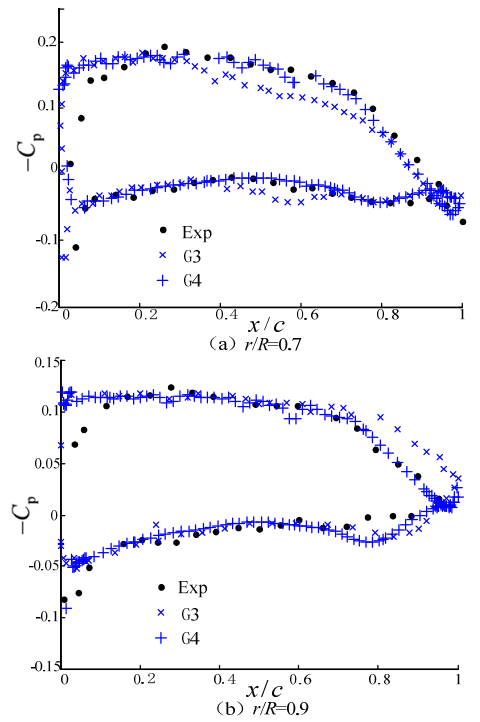

Figure 7: $\quad$ Pressure coefficient distribution at different sections.

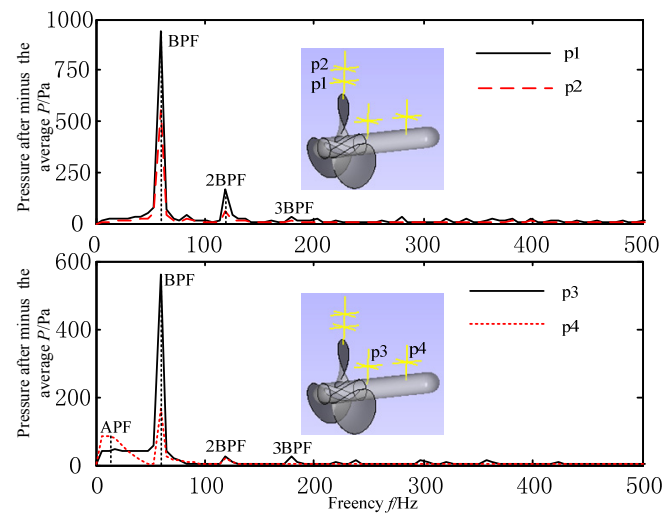

Figure 8: $\quad$ Fluctuated pressure spectrum for unsteady flow field.

After a steady solution has been computed, to be sure that the flow is really steady, an unsteady simulation should be carried out with the existing steady flow field as the initial condition. What's more important, the subsequent transient simulation is used to get the fluctuated source. The time step corresponding to 2 degrees of propeller rotation is $\Delta t=2.78 \times 10^{-4} \mathrm{~s}$, and the total time is $0.15 \mathrm{~s}$ corresponding to 3 circles. The last week's transient results are used to extract the pressure and normal velocity used in equation (4). In the process of unsteady simulation, special monitor points are set to get the time series pressure which can determine the validity of the calculation indirectly in terms of the fluctuating information. Four points are seen in Fig. 8. The calculated the 
pressure amplitude with the FFT transformation after minus the average value is shown in Fig. 8. The monitors are placed on radial and axial direction of the blades. The results reveal that the fluctuated pressure of both of the point $\mathrm{p} 1$ and p2 can represent the blade passing frequency (BPF) and its harmonics information due to the rotating of blades, and the amplitude will decrease with the radial distance increase. As for the axial point $\mathrm{p} 3$ which is relative near to the blades, it is still sensitive to the rotating effect. And both of its BPF and harmonics appear again. When the point shifting downstream to the p4 point, the propeller wake effect weakens, the amplitude of BPF decreases rapidly, and the axial passing frequency (APF) information presents at this time.

\subsection{Acoustic prediction}

After the load noise source term according to equation (2) has been solved, the fluctuated pressure in time domain on the blades is transformed to the acoustic nodes using one to one model within frequency domain. Fig. 9 shows the blades pressure distribution in frequency domain obtained by the DBEM equation. The tip area and trailing edge near the root section contribute mainly to the noise under the low frequency. Specifically, the tip vortex core resulting low pressure and the trailing edge vortex shedding as the noise source take a dominating effect. Wherein, for the relatively high frequency, the noise highlight area shifts to the load centered region on the blades wall, and the load noise is the main noise source at this time.

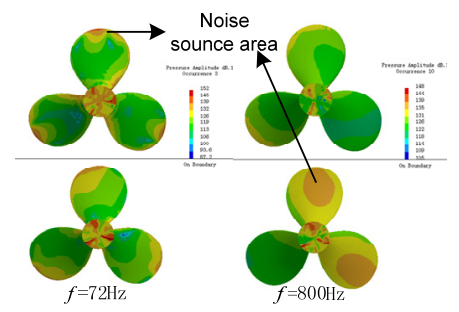

Figure 9: Blades pressure distribution in frequency domain.

To be consistent with the prediction in references [4] and [5], the noise directivity characteristics on the vertical plane along the axis and the sound pressure level spectrum in the same broadband frequency on given field point in the wake flow of 4119 propeller within spatial non-uniform inflow are analyzed. The calculated sound pressure distribution on axial vertical circinal-plane with the radius of $10 R$ is seen in Fig. 10. In the low frequency broadband, the load noise presents obvious axial dipole information, the acoustic energy concentrated with a strong radiation tendency towards the observer on the hub axis. Within the high frequency, the dipole nature of the acoustic source also presents, but the source concentrated area enlarging with a deflection to the axial direction, and the slope angle depends on the frequency and inflow velocity. If the field points series consist of a three-dimensional spherical surface, on where the sound pressure distribution in the low frequency is seen in Fig. 11 with a qualitative 
agreement with and the calculated results of reference [5] because of the unawareness of exact frequency under $200 \mathrm{~Hz}$ in that. Fig. 12 shows the sound pressure spectrum on the axial point downstream with a distance of 10 times the propeller radius. Because the ship wake used in this paper is not the same as that in references, the tendency and quantitative agreement with the reference results just can be obtained. From Figs. 11 and 12, it can interpret indirectly the availability of the coupling method given in this paper to predict the underwater radiated noise of ship propellers.

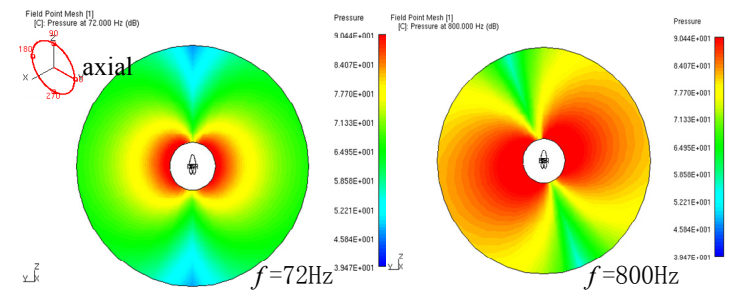

Figure 10: Sound pressure distribution on the plane.

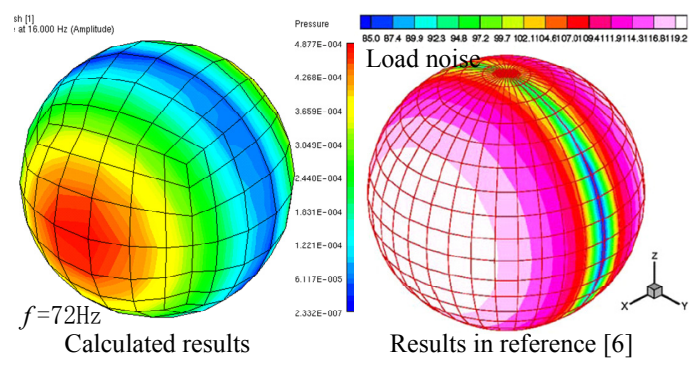

Figure 11: Sound pressure distribution on spherical surface.

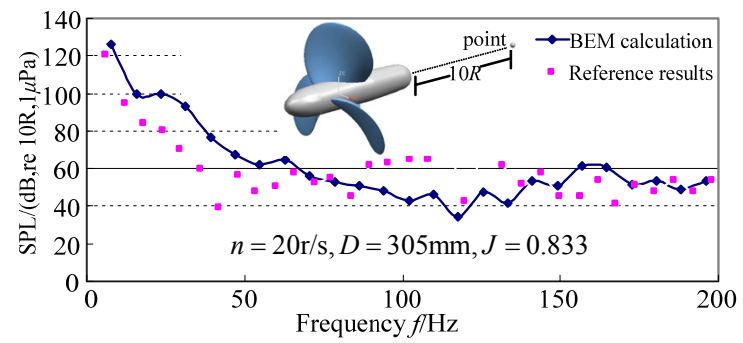

Figure 12: Sound pressure spectra on visual measured point.

The overall sound pressure level of the visual measured point calculation according to the equation

$$
S P L_{\text {total }}=10 \log \left(\sum_{i=1}^{i=n} 10^{0.1 S P L(i)}\right)
$$


is $126.4 \mathrm{~dB}$, where the $S P L(i)$ is the $S P L$ in each one-third Octave band to the equivalent $1 \mathrm{~Hz}$ bandwidth by means of the correction formula accounting for the frequency broadband.

\section{Conclusions}

This paper presents the weak coupling LES and BEM numerical acoustics method in frequency domain to predict the underwater radiated load noise of DTRC4119 propeller. The sound strength distribution on the blades surface, sound pressure distribution on the axial vertical plane and spatial spherical surface and the broadband spectra of the measured point downstream are presented and compared with that in references. The calculated steady flow field is fit well with the experiment data. The unsteady simulation can precisely present the fluctuated BPF and its harmonics information. The predicted spatial sound pressure distribution and the field point's noise level spectra are both reasonable with the tendency and quantity are the same as that in references, which can indicate the availability of this coupling method to predict the propeller's underwater radiated noise in engineering.

\section{Comments}

The analyzed condition given in references [4] and [5] is $n=120 \mathrm{rpm}, v=1.6 \mathrm{~m} / \mathrm{s}$. But the diameter D is unknown. For the generally propeller model's open water experiment and CFD analysis, the D is about 0.25 to 0.35 meters, and the ITTC report gives $\mathrm{D}=0.305 \mathrm{~m}$ for the 4119 propeller. So the advance ratio $J=v /(n D)$ is 2.29 to 3.2 , lying in the fourth quadrant, and the thrust coefficient is negative which is impossible for analysis. On the other hand, as the design point of 4119 propeller is $J=0.833$, if the rotating speed is right, the $\mathrm{D}$ will be $0.96 \mathrm{~m}$, which is nearly to the full scale. Nowadays, even the successful viscous CFD calculation, the prediction error will be about $4 \sim 5 \%$ for the full scale, and let alone the nonviscous panel method. Hence, for the promising fluctuated source prediction, the $n$ will be 20 rps as same as the commonly used in the hydrodynamics.

\section{References}

[1] Carlton J S. Marine propellers and propulsion. Second Edition. Elsevier Ltd.: Netherlands, pp. 240-283, 2007.

[2] Caridi D. Industrial CFD simulation of aerodynamic noise. Ph.D dissertation, University Degli Studi Di Napoli Federico II, 2008.

[3] Ross D. Mechanics of underwater noise. Pergamon Press: New York, 1976.

[4] Seol H, Jung B, Suh J C, et al. Prediction of non-cavitation underwater propeller noise. Journal of Sound and Vibration, 257(1), pp. 131-156, 2002.

[5] Seol H, Suh J C, Lee S. Development of hybrid method for the prediction of underwater propeller noise. Journal of Sound and Vibration, 288, pp. 345-360, 2005. 
[6] Carlton J S. Marine propellers and propulsion. Second Edition. Elsevier Ltd.: Netherlands, pp. 435-438, 2007.

[7] Hu Jiang. Research on propeller cavitation characteristics and low noise propeller design. Ph.D thesis, University of Harbin Engineering, 2006.

[8] Kato C, Yamade Y, Wang $\mathrm{H}$, et al. Numerical prediction of sound generated from flows with a low mach number. Computers \& Fluids, 36, pp. 53-68, 2007.

[9] Testa C, Acoustic formulations for aeronautical and naval rotorcraft noise prediction based on the Ffowcs Williams and Hawkings equation. Ph.D dissertation, Delft University of Technology, 2008.

[10] Carley M, Time domain calculation of noise generated by a propeller in a flow. Ph.D dissertation, Department of Mechanical Engineering, Trinity College, Ireland, 1996.

[11] Wang M, Freund J B, Lele S K. Computation prediction of flow-generated sound. Annual Review of Fluid Mech, 38, pp. 483-512, 2006.

[12] LMS Inc. BEM acoustics. LMS Virtual Lab online Help, 2006.

[13] LMS International. Numerical Acoustics. LMS Virtual Lab Theoretical Manual, 2006.

[14] Kobayashi T. Large eddy simulation for engineering applications. Fluid Dynamics Research, 38, pp. 84-107, 2006.

[15] Holm D. D., Geurts B. Commutator errors in large-eddy simulation. Journal of Physics A: Mathematical and General, 39, pp. 2213-2229, 2006.

[16] Vysohlid M. Large eddy simulation of crashback in marine propellers. Ph.D dissertation, University of Minnesota, 2007.

[17] Escobar M. Finite element simulation of flow-induced noise using Lighthill's acoustic analogy. Ph.D thesis, University Erlangen-Nurnberg, 2007.

[18] Yang Qiongfang, Wang Yongsheng, GUO Wei et al. Procedural realization of pre-operation in CFD prediction of propeller hydrodynamics. Accepted by Journal of Ship Mechanics, 2010 (in Chinese).

[19] Yang Qiongfang, Wang Yongsheng, Huang Bin et al. Integrated lifting line theory and RANS simulation for propeller design and non-cavitation and cavitation hydrodynamics prediction. Accepted by Journal of Shanghai Jiaotong University, 2010 (in Chinese).

[20] Yang Qiongfang, Wang Yongsheng, Fan Lu. Hydrodynamic performance analysis and optimization design of mechatronic ducted propeller with CFD. Chinese Journal of Mechanical Engineering, 46(1), pp. 162-168, 2010. 